\title{
Real World Evidence and the Behavioral Economics of Physician Prescribing
}

\author{
Garza Samantha
}

Cardinal Health Specialty Solutions 7000 Cardinal Place, Dublin, OH 43017, USA

*Corresponding author: Cardinal Health Specialty Solutions 7000 Cardinal Place, Dublin, OH 43017, USA, Tel: +614-757-9015; E-mail: samantha.garza@cardinalhealth.com

Received date: August 01, 2016; Accepted date: August 29, 2016; Published date: September 06, 2016

Copyright: ( 2016 Samantha G. This is an open-access article distributed under the terms of the Creative Commons Attribution License, which permits unrestricted use, distribution and reproduction in any medium, provided the original author and source are credited.

\section{Commentary}

The projections for the rising cost of health care have spurned robust dialogue from every sector of the healthcare economy $[1,2]$ Among the many targets for cost control are specialty drugs distinguished clinically by their route of administration, synthesis or bioengineering, mechanism of action and cost itself [2]. This terminology likely originated from payers who designate these drugs for special attention not only because of price, but also the need for distinctive handling or particular patient monitoring [3]. Although there are examples of competition emerging to tamp down prices to more acceptable levels (e.g. pharmacy benefit manager negotiations for hepatitis C drugs), stakeholders (policy-makers, insurance carriers, and non-governmental groups such as ASCO) are seeking other market-based solutions [2].

The fee-for-service payment system has been identified as one of the main drivers of rising health care costs the more that is done for patients, the larger the reimbursement to the healthcare provider [1]. In the fee-for-service model, providers may directly purchase the drugs they administer to patients in their in-office infusion suites from manufacturers and/or wholesalers then bill the payer for cost plus margin [4,5] Many argue that this "buy-and-bill" model encourages physicians to overprescribe, creates incentives for price inflation and thereby drives up the costs of patient care [6]. A few studies have even suggested that providers' choice of drug treatment can be affected by reimbursement, resulting in their over-utilizing more costly brands rather than less expensive brand or generic alternatives [7]. Such suggestions draw the ire of providers who believe the portrayal of patient care as solely driven by financial incentives and behavioral economics is insulting, demeans their professional integrity, and is inconsistent with real world evidence that demonstrates highly variable regional resource utilization as well as few differences in prescribing patterns between community, staff model and academic physicians when controlling for these geographic variances [8].

In their editorial in NEJM, Khullar et al, outline the basic tenets of behavioral economics established by Kahneman et al.: behavioral economics views incentives as fundamental determinants of behavior; people often make decisions that deviate from those expected of "rational" economic actors [9]. By their assessment most behavioral economics work in healthcare has focused on those factors influencing patients' behavior with less attention paid to applying behavioral economics to understanding physicians' performance and decision making [9]. Behavioral economists have identified certain patterns in their observations of economically "irrational" behaviors a few of which merit attention as they are germane to healthcare provider decision making:

Heuristics: people often make decisions based on approximate rules of thumb and not strict logic.
Framing: the collection of anecdotes and stereotypes that make up the mental and emotional filters individuals rely on to understand and respond to events.

Defaults: a preference for continuing the current state of affairs or status quo.

Loss Aversion: losses have about twice the psychological impact of commensurate gains.

These theories, experimentally tested and validated, not only explain financial decision making but likely much of decision making in general. Physicians, being human, are subject to irrational behaviors in their professional decision making just as they are in any aspect of their life. Pursuant to this logic the practice of evidence based medicine is likely to be significantly impacted by these realities of human thought and behavior. This helps explain the need for both heavily regulated clinical trial research and for real world evidence to understand how such trial results are adopted into everyday practice. My research the past five years has focused on exactly this topic, the variances between clinical trial research and real world evidence and the behavioral economics that underscore them.

Much of this research emerged from my work with collaborative clinical pathways programs between insurers and their specialty physician provider networks. In partnership with one large non-profit healthcare insurer in the Mid-Atlantic region of the United States, the first network-wide, collaborative, cancer clinical pathway was launched in August 2008 [10,11]. Due to high levels of physician participation, compliance and behavior change, the adoption of the pathway resulted in a $15 \%$ cost of care reduction and led to improved patient outcomes including a $7 \%$ reduction in ER visits and hospitalizations $[11,12]$. To test the further impact of reimbursement reform, a second-generation pathways program-the Oncology Medical Home was piloted in 2011 [13]. This program modified the traditional physician reimbursement model used in the pathway program shifting the source of revenue from drug reimbursement margin to enhanced professional charges for cognitive services (evaluation and management codes E\&M) [13]. The medical home removed financial incentive from drug delivery while dramatically increasing it for patient care.

A select group of practices that participated in the first-generation pathways program were invited to voluntarily participate in the Oncology Medical Home. The intent was to compare physician behavior parameters pre- and post-implementation and between the Oncology Medical Home practices and the first-generation pathways control group [13]. The methodology of conducting research in such circumstances is difficult; by definition, selection bias exists when programs are voluntary and financially incentivized [13]. However, any selection bias incurred impacted both control and experimental cohorts, which was controlled for in analysis by propensity score matching using the following variables: disease focus, diagnosis mix, 
number of heavily treated versus newly treated patients, early stage versus later-stage treatment, total chemotherapy lines ("extent of treatment"), and patient comorbidities [13,14]. Taken together, we believe these measures yielded propensity scores indicative of case mix.

Although I take pains to explain the quality of the research this is a separate issue from the main question of whether physicians behave to maximize their financial gain. The behavioral economic incentives designed within the model predicted that the medical home physician participants would increase the frequency of patient visits and likely decrease the per cent of patients undergoing chemotherapy treatment as well an increase the use of generic drug in those who did. Surprisingly, physician behavior was not significantly modified by the cognitive weighted reimbursement model [13]. This research, which we published in The American Journal of Managed Care, revealed: no significant change in the frequency of office visits for established patients; no change in the pattern or choice of chemotherapy prescribing; and observed increases in generic regimen use were no different than that of the matched control [13].

How do we reconcile these findings and the prevailing dogma that resulted in a 2014 New York Times editorial co-signed by 20 leading academics citing fee-for-service reimbursement as the primary driver for the spiraling cost of cancer care in this country? [6] What are the behavioral economic explanations for the economically irrational pattern of care we observed? To what extent did the prior pathway experience influence behavior? Were the observed behaviors driven by a mix of other factors including: National Comprehensive Cancer Network and other guidelines (default), brand name prescription drug detailing (attribute priming), cognitive dissonance (heuristics), or our culture of medicine (framing)? Without more detailed research, any conclusions can only be speculative [13].

Additionally, the results from this study are in contradiction to one of the more powerful behavioral economic incentives, loss aversion. The participating physicians on average experienced an annualized $2 \%$ reimbursement reduction, and despite a nearly 3-fold increase in E\&M code reimbursement, no significant change in established patient visits was observed to mitigate said loss. This was contrary to expectation and could be related to external influences on physician practice behavior (framing), including the historically lower contribution of E\&M reimbursement to revenue (heuristics), standardized and established practice patterns (default), and maximized throughput within office flow (heuristics) [13].

We believe our research was methodologically sound, adequately powered and the results unequivocal: given the opportunity to maximize revenue by increasing select cognitive services, physicians remained unchanged in their behaviour [13]. Furthermore, removing economic incentives from drug prescribing did not alter the pattern of prescribing. Our research should make economic Darwinists take heed as the bedrock on which much of reimbursement reform is founded assumes more rational economic behavior. The myriad factors that weigh on physician prescribing behavior for complex, disabling and potentially life threatening diseases like Cancer, Multiple Sclerosis, Crohn's and a host of inflammatory and infectious diseases, as well as those factors impacting the patients receiving the treatment, eclipse the most sophisticated of behavioral economic modeling. In this context, the speculated impact of reimbursement reform may be overestimated. Observations from this oncology medical home pilot program suggest that reimbursement methodology alternatives to the prevailing fee-forservice may have less impact on prescribing behavior than has been conjectured [13]. Future research is needed to validate these observations and assess additional influences on prescribing behavior. It may be prudent to defer radical reimbursement reform until such research is conducted and results subjected to peer review.

\section{References}

1. Smith TJ, Hillner BE (2011) Bending the cost curve in cancer care. N Engl J Med 364: 2060-2065.

2. Feinberg B, Lal LS, Swint JM (2015) Is there a mathemetical resolution to the cost-versus-value debate? Am J Manag Care 21.

3. Kesselheim AS, Avorn J, Sarpatwari A (2016) The high cost of prescription drugs in the United States origins and prospects for reform. JAMA 316: 858-871.

4. Sprandio JD (2012) Oncology patient-centered medical home. J Oncol Pract 8: 47s-49s.

5. Jacobson M, Earle CC, Price M, Newhouse JP (2010) How Medicare's payment cuts for cancer chemotherapy drugs changed patterns of treatment. Health Aff (Millwood) 29: 1391-1399.

6. Emanuel EJ (2013) A plan to fix cancer care. New York Times SR14.

7. Jacobson M, O'Malley AJ, Earle CC, Pakes J, Gaccione P (2006) Does reimbursement influence chemotherapy treatment for cancer patients? Health Aff (Millwood) 25: 437-443.

8. Gottlieb DJ, Zhou W (2010) Prices do not drive regional medicare spending variations. Health Aff March 29: 537-543.

9. Khullar D, Chokshi DA, Kocher R, Reddy A, Basu K, et al. (2015) Behavioral economics and physician compensation--promise and challenges. N Engl J Med 372: 2281-2283.

10. Feinberg B, Lang J, Grzegorczyk J (2012) Implementation of cancer clinical care pathways: A successful model of collaboration between payers and providers. Am J Manag Care 18: e194-e199.

11. Feinberg B, Milligan S, Cooper J (2013) Third-party validation of observed savings from an oncology pathways program. Am J Manag Care 19.

12. Kreys ED, Koeller JM (2013) Documenting the benefits and cost savings of a large multistate cancer pathway program from a payer's perspective. J Oncol Pract 9: e241-e247.

13. Feinberg B, Milligan S, Olson T, Wong W, Winn D, et al. (2014) Physician behavior impact when revenue shifted from drugs to services. Am J Manag Care 20: 303-10.

14. Austin PC (2011) An introduction to propensity score methods for reducing the effects of confounding in observational studies. Multivariate Behav Res 46: 399-424. 